\title{
Utility of Alternative Ankle Brachial Pressure Index for Screening Asymptomatic Peripheral Arterial Diseases in Patients with Acute Myocardial Infarction and Cerebrovascular Accident
}

\author{
Amulya Cherukumudi ${ }^{1}$, Kalale Radhakrishna Iyengar Bhagavan ${ }^{2}$ \\ 1,2 Department of General Surgery, K.S. Hegde Medical Academy, Mangalore, Karnataka, India.
}

\section{ABSTRACT}

\section{BACKGROUND}

Peripheral arterial disease (PAD) is one of the macrovascular manifestations of systemic, diffuse atherosclerosis. Patients with acute myocardial infarction are at 3 5 times higher risk of developing associated PAD. Ankle Brachial Pressure Index (ABPI) is a simple, non-invasive test to diagnose peripheral arterial diseases in symptomatic cases. We wanted to assess the utility of an alternative formula for Ankle Brachial Pressure Index as a sensitive screening tool for asymptomatic peripheral arterial diseases in patients with acute myocardial infarction and cerebrovascular accident.

\section{METHODS}

This is a prospective observational study, recruiting eligible patients from medicine and cardiology outpatient department (OPD). Patients included were those with acute myocardial infarction (MI) or cerebrovascular accident (CVA). Sample size was calculated to be 257. ABPI was measured using a hand-held Doppler of $8 \mathrm{MHz}$ and a standard sphygmomanometer. Statistical analysis was performed using SPSS software.

\section{RESULTS}

Alternate ABPI showed a mean value of $0.9+/-0.072$. In the study population, 61.1 $\%$ were found to have abnormal ABPI i.e., less than 0.9 , which is nearly two thirds of the population. Receiver operating characteristic curve (ROC) was plotted against ABPI for sensitivity and specificity for the co-morbidities considered in the study. It was found that the AUC (Area Under the Curve) was statistically significant for acute MI and acute CVA, with a fair positive correlation.

\section{CONCLUSIONS}

The alternative, ABPI is a good screening test for detecting asymptomatic PAD in patients with acute MI and CVA. However, further comparative studies are required to confirm the utility of the same in such patients, especially in a larger, varied population.

\section{KEY WORDS}

Ankle Brachial Pressure Index, Atherosclerosis, Peripheral Arterial Disease, Cardiovascular Diseases
Corresponding Author:

Dr. Kalale Radhakrishna Iyengar Bhagavan, Department of General Surgery,

K.S Hegde Medical Academy,

Mangalore - 575018, Karnataka, India.

E-mail: krbhagavan@rediffmail.com

DOI: $10.14260 /$ jemds/2021/147

How to Cite This Article:

Cherukumudi A, Bhagavan KRI. Utility of alternative ankle brachial pressure index for screening asymptomatic peripheral arterial diseases in patients with acute myocardial infarction and cerebrovascular accident. J Evolution Med Dent Sci 2021;10(10):684-688, $10.14260 /$ jemds/2021/147

Submission 26-10-2020,

Peer Review 10-01-2021,

Acceptance 16-01-2021,

Published 08-03-2021.

Copyright (C) 2021 Amulya Cherukumudi et al. This is an open access article distributed under Creative Commons Attribution License [Attribution 4.0 International (CC BY 4.0)] 


\section{BACKGROUND}

Peripheral arterial disease is one of the macrovascular manifestations of systemic, diffuse atherosclerosis. Patients can be asymptomatic or have symptoms of claudication pain, gangrene and myopathy. Patients with acute myocardial infarction are at 3 - 5 times higher risk of developing associated PAD. ${ }^{1}$ The rationale for screening to detect patients with asymptomatic peripheral artery disease is based upon the below possible objectives. ${ }^{2}$

- To identify early PAD and intervene to prevent progression and complications directly related to PAD.

- To identify patients at high risk for PAD with underlying cardiovascular diseases

- To achieve the first objective, the risk of progression from asymptomatic PAD to critical leg ischemia should be sufficiently high to warrant intervention in individuals identified by screening, the interventions should be safe and have proven effectiveness in preventing progression of PAD, and the interventions should be those that would not be implemented without screening.

The US Preventive Services Task Force (USPSTF) found that the existing evidence in inadequate to determine whether screening of asymptomatic PAD is beneficial to the patient in terms of preventing further progression or in terms of preventing other cardiovascular events such as CAD or CVD. ${ }^{3}$ The target population involves those individuals aged 50 or more, with a positive history of smoking (corresponds to the PAD guidelines of the American College of Cardiology Foundation (ACCF) / American Heart Association (AHA) Task Force).

The various methods for detecting PAD recommended for such asymptomatic patients can be further classified as ${ }^{3}$

- Invasive such as contrast angiography, magnetic resonance angiography, computed tomographic angiography and photo plethysmography

- Non-invasive techniques include clinical history, physical examination (symptoms of leg pain, palpation of peripheral pulses), exercise test and questionnaires (Edinburgh Claudication questionnaires), ABPI, ToeBrachial Index (TBI), Duplex scan of the peripheral circulation and pulse oximetry.

In clinical practice, several tools exist to diagnose PAD in patients with symptoms for the same. ABPI is the ratio of the systolic blood pressure (BP) in the arm to the systolic $\mathrm{BP}$ in the leg. The rationale behind ABPI is that a reduced peripheral flow in comparison to the central circulation, we can make a diagnosis of stenosis of that vessel. ABPI is a simple, noninvasive test to diagnose peripheral arterial diseases in symptomatic cases. When the ABPI is less than 0.9 , it is considered abnormal; less than 0.5 is indicative of critical limb ischaemia. However, utility of ABPI in asymptomatic cases has the added advantage of guiding the clinician in deciding the further imaging modality and treatment that the patient might require.

1) As part of clinical examination, history of claudication is essential to record. However, when compared with ABPI, it has low sensitivity and PPV, which makes it a poor screening method.
2) During general physical examination, palpation of the distal peripheral pulses (dorsalis pedis artery, posterior tibial artery and anterior tibial artery), popliteal artery and femoral artery pulses plays an essential role in the screening of PAD in high-risk patients. Apart from this, shiny skin over the lower limbs, presence of dilate tortuous veins, pallor of elevation on limb, gangrene or ulceration and loss of hair can point towards a critical limb ischaemia (CLI). ${ }^{4}$ Other findings such as ulceration, loss of hair or shiny skin may indicate critical limb ischaemia. However, such findings for the purposes of screening, have low sensitivity when compared to ABPI. ${ }^{5}$

3) Exercise / walking tests help to evaluate the extent of limitation of function in a limb afflicted by chronic limb ischaemia, and it also helps in differentiating it from other conditions that may be aggravated or relieved by exercise. ${ }^{5}$ The walking tests essentially is to check the claudication distance, and this is done by making the patient walk at a pre-fixed velocity. The ABPI measured once prior to the onset of the test, and once immediately after the test. ${ }^{5}$ In patients without PAD, a slight increase is observed in the ABPI when compared to the pre-test readings. When a patient with $\mathrm{PAD}$ develops claudication or decrease in the ankle pressure as compared to the pretest reading, an arterial occlusion is suspected. A reduction in ankle systolic blood pressure (SBP) by $>20$ $\%$ or less than $60 \mathrm{~mm} \mathrm{Hg}$ is considered abnormal. ${ }^{6}$

4) Several questionnaires are available as a screening method for PAD - Edinburgh Claudication Questionnaire (ECQ) or the World Health Organization (WHO) / Rose questionnaire. The ECQ is a modified format of the WHO / Rose questionnaire. It is observed that the ECQ as a screening tool has a high sensitivity and specificity when compared to history taking alone. However, this is useful only in those with intermittent claudication. It is not apt for asymptomatic patients, unlike ABPI.

5) ABPI is a simple, non-invasive methods to screen for and diagnose underlying PAD, and risk-stratify patients that are at a high risk for cardiovascular events. It is a simple ratio of the systolic pressure of the lower limb with the upper limb, and it requires the use of a hand-held Doppler probe, making it non-invasive and acceptable to patients for screening. ${ }^{7}$ The methods of ABPI measurement has not been standardised, and hence, several methods have been suggested for the same. According to the UK NICE clinical guidelines, patient is placed in a supine position, and the respective ankle and brachial systolic pressures are measured with the Doppler probe (posterior tibial artery for ankle SBP and brachial artery for brachial SBP). The cuff is tied just above the ankle, after the patient is made to lie down for a duration of 15 - 30 minutes. The cuff is insufflated above the pressure at which the Doppler sound wave is not audible, and slowly reduced. The point at which the sound is audible again, is taken as the ankle SBP. The same procedure is done for the opposite limb and well as bilateral upper limbs. ${ }^{8-10}$ This approach provides a reasonable estimate of the potential for healing foot wounds and may be less insensitive for detecting PAD. This is important to keep in mind when using ankle pressures as a means to screen asymptomatic patients for PAD; using the highest ankle pressure may fail to identify patients who would benefit from risk factor 
modification or medical intervention to reduce the overall cardiovascular morbidity and mortality. An alternative method for screening uses the lower two ankle pressures as the numerator in the ratio.

6) In this present study, we aim to assess the utility of an alternative formula for ABPI as a sensitive screening tool for asymptomatic peripheral arterial diseases in patients with acute myocardial infarction and cerebrovascular accident.

\section{METHODS}

The study was conducted as a prospective observational study, after institutional ethical committee clearance (IEC NUMBER INST.EC / EC / 111 / 2017 - 18). The study participants were aged between 18 - 80 years, and those with previous acute myocardial infarction (diagnosed within 6 months of first attack) or acute cerebrovascular accident (diagnosed within 6 months of the first attack), admitted from medicine or cardiology OPD between October 2017 to June 2019. The days of admission were randomised by lottery method and only patients admitted on Tuesday and Thursday in Justice K.S. Hedge Charitable Hospital were included in the study to eliminate selection bias. The sample size was calculated based on a study done by Mourad J.J. et al. which was found to be 257 at $95 \%$ confidence interval.

\section{Sample Size Calculation}

In a study conducted by Mourad J-J, Cacoub P, Collet J-P, Becker F, Pinel J-F, Huet D, et al. ${ }^{11}$ in individuals with unrecognised peripheral arterial disease using ankle-brachial index in high cardiovascular risk patients free from symptomatic PAD was $41.1 \%$. Expecting similar results, at $95 \%$ confidence interval, $5 \%$ relative precision, a minimum sample of 257 patients would be required assuming that about 500 patients with high cardiovascular risk patients would be visiting the hospital during the study period. This was calculated using formula

$\mathrm{n}=\frac{(4 \times \mathrm{p} \times \mathrm{q})}{\mathrm{d}^{2}}$

where $p$ is expected true proportion, $q$ is $(1-p)$, and $d$ is desired precision.

Adjustment for finite population was done using formula$n(\operatorname{adj})=\frac{(N \times n)}{(N+n)}$

Those patients with symptomatic peripheral arterial disease or with gangrenous changes, chronic kidney disease, post-menopausal women and pregnant women were excluded from the study as these are confounding factors. Institutional ethical committee clearance was sought and granted for the purposes of this study. The consent to conduct the study was obtained from the hospital authorities.

Once informed consent was sought, patient was made to lie in supine position after a duration of rest of half an hour. Peripheral pulses were palpated (posterior tibial artery for lower limb and brachial artery for upper limb. The pre- requisite for our study was that patients have a palpable peripheral pulse. Once this was confirmed, Ankle Brachial Pressure Index was measured. A standard hand-held Doppler with a probe of $8 \mathrm{MHz}$ was used, with a standard sphygmomanometer. ABPI was indicative of PAD if less than or equivalent to 0.90 and normal if more than or equal to 1.00 .

We utilised an alternative formula for ABPI measurement, which is mentioned below-

- $\quad$ ABPI (alternative) - SBP of ankle (lower of the two limbs) / SBP of the brachial (lower of the two limbs)

- The demographic details, clinical examination findings and measured ABPI values (standard formula was also calculated) was entered in a semi-structured pro forma.

\section{Statistical Analysis}

The data was then entered in a spreadsheet of MS Excel. The statistical analysis was performed using SPSS software v20. ROC curve for ABPI utility for each co-morbidity (acute MI and CVA) was plotted separately, and the area under the curve was measured for sensitivity and specificity of ABPI as a screening tool for PAD.

\section{RESULTS}

Total number of patients included in the study was 257, of whom $202(78.6 \%)$ were males. Majority of the patients belonged to 41 - 50 years age group (35.4\%) (Figure 1).
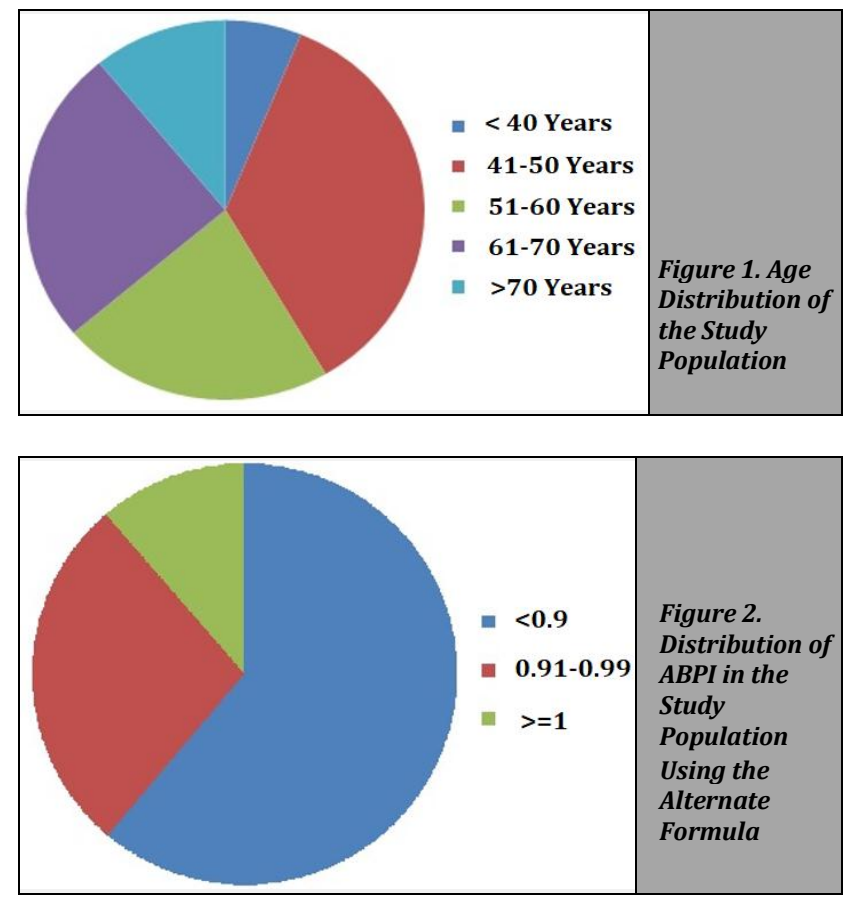

\begin{tabular}{|cc|}
\hline ABPI & Frequency (\%) \\
$\leq 0.9$ & $157(61.1)$ \\
$0.91-0.99$ & $71(27.6)$ \\
$\geq 1.0$ & $29(11.3)$ \\
Total & $257(100.0)$ \\
\hline \multicolumn{2}{|c|}{ Table 1. ABPI Classification (Using Alternate Formula) } \\
in the Study Population
\end{tabular}




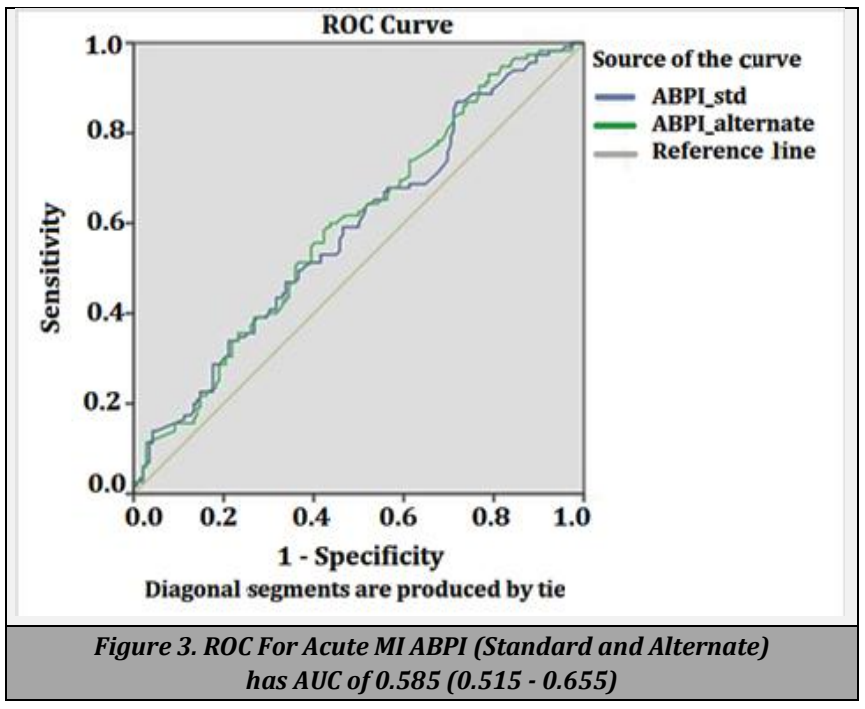

\begin{tabular}{|cc|}
\hline Sensitivity & 0.61 \\
Specificity & 0.44 \\
Positive predictive value & 0.59 \\
Negative predictive value & 0.76 \\
Table 2. Sensitivity and Specificity of ABPI in Acute MI
\end{tabular}

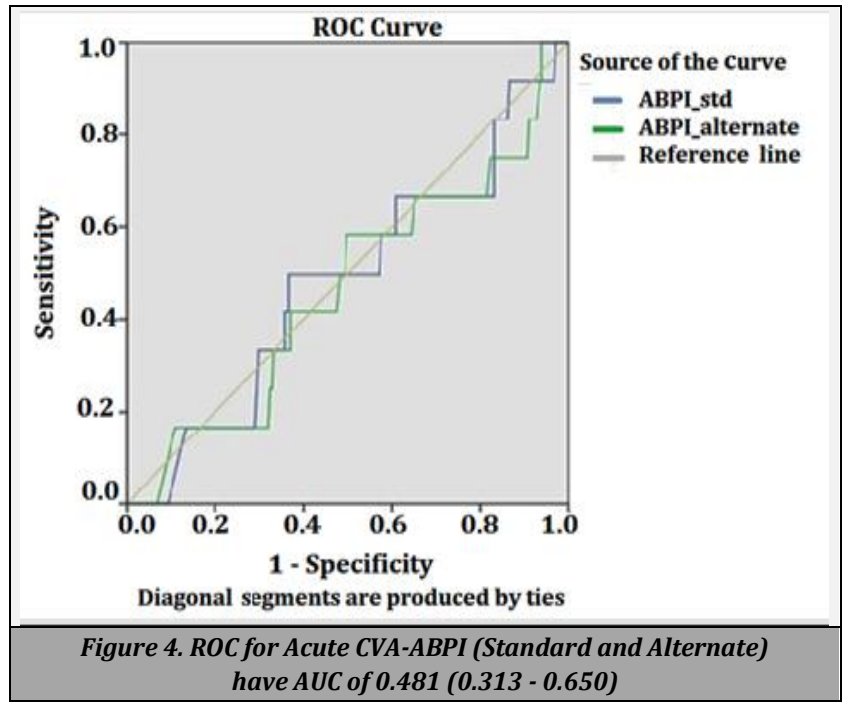

\section{DISCUSSION}

Peripheral arterial disease is a common vascular complication of atherosclerosis, causing significant morbidity and mortality; can further increase the risk of myocardial infarction and cerebrovascular accidents. It is estimated that about $68 \%$ of patients with PAD also are incidentally diagnosed with coronary artery disease; ${ }^{2}$ the corollary is also true. The reason for co-existence of PAD with CAD and CVA is due to the fact that atherosclerosis is a systemic disease and can affect arteries of any calibre.

It has been studied that the lower the ABPI value, the higher is the risk for acute MI and acute CVA. In our study, we observed that almost 2 / 3 rd the study population showed an abnormal ABPI value. Patients with PAD are 6 times more like to succumb to CAD or CVA than those that do not have the disease. ${ }^{7}$ Hence, it is essential to diagnose PAD in such highrisk patients, to prevent such mortality.
In a consensus conference report, ABPI was defined as the ratio of the higher systolic blood pressure in the anterior tibial / posterior tibial artery to the average of the right and left brachial arteries. If the difference between the right and the left brachial arteries was more than $10 \mathrm{mmHg}$, then the higher of the two values is taken.7,8 However, recent American Heart Association guidelines doesn't clearly define whether the higher or lower of the two ankle pressures are to be used. This along with the inter-observer variability and improper technique is the reason that there are several inconsistencies in the ABPI values. Hence, a standardised formula for the same is necessary to diagnose accurately the underlying PAD, especially in asymptomatic patients in high-risk cases such as those with acute MI and acute CVA, as in our study. ${ }^{10-12}$

In our study, we considered the lower of the two systolic blood pressures of both ankle and brachial, the "alternate" ABPI. In our study we found that there was a positive correlation between "alternate" ABPI values and the presence of acute MI or CVA. This depicts the role of "alternate" ABPI as a fair screening tool in such high-risk cases. A study done by Shroeder et al. suggested a formula merging the standard and alternate formula and found that it demonstrated a higher sensitivity for screening asymptomatic PAD.12-14 In a study conducted by Niazi $\mathrm{K}$ et al. they demonstrated that the "alternate" ABPI has a higher sensitivity than the standard ABPI. ${ }^{15}$ This variation could eliminate any changes that may be induced in the blood vessel due to calcification causing thickening. Also, by considering the lower of the two values, we can also possibly eliminate the effect of systemic hypertension that may co-exist in such high-risk cardiovascular patients. ${ }^{16-18}$

\section{CONCLUSIONS}

ABPI is a fair screening tool for identifying PAD in asymptomatic yet high risk patients with previous history of acute MI and acute CVA. Further studies are required to compare the findings with Duplex scan or computed tomography (CT) angiography to confirm the validity of the same.

\section{Limitations}

Several patients in our study were diabetics, which could be the cause of medial calcinosis of vessels, causing underestimation of the incidence of PAD in the study population.

Data sharing statement provided by the authors is available with the full text of this article at jemds.com.

Financial or other competing interests: None.

Disclosure forms provided by the authors are available with the full text of this article at jemds.com.

\section{REFERENCES}

[1] Saw J, Bhatt DL, Moliterno DJ, et al. The influence of peripheral arterial disease on outcomes: a pooled analysis of mortality in eight large randomized percutaneous coronary intervention trials. J Am Coll Cardiol 2006;48(8):1567-72. 
[2] Hirsch AT, Haskal ZJ, Hertzer NR, et al. ACC / AHA 2005 practice guidelines for the management of patients with peripheral arterial disease (Lower extremity, renal, mesenteric and abdominal aortic): a collaborative report from the American association for vascular surgery / society for vascular surgery, society for cardiovascular angiography and interventions, society for vascular medicine and biology, society of interventional radiology, and the ACC / AHA task force on practice guidelines (writing committee to develop guidelines for the management of patients with peripheral arterial disease): endorsed by the American Association of cardiovascular and pulmonary rehabilitation; National Heart, Lung and Blood Institute; Society for vascular nursing; Trans Atlantic Inter-Society Consensus; and vascular disease foundation. Circulation 2006;113(11):e463-654.

[3] Alahdab F, Wang AT, Elraiyah TA, et al. A systematic review for the screening for peripheral arterial disease in asymptomatic patients. J Vasc Surg 2015;61(Suppl 3):42S-53S.

[4] Guirguis-Blake JM, Evans CV, Redmond N, et al. Screening for peripheral artery disease using the ankle-brachial index: updated evidence report and systematic review for the US preventive services task force. JAMA 2018;320(2):184-96.

[5] Lyu X, Li S, Peng S, et al. Intensive walking exercise for lower extremity peripheral arterial disease: a systematic review and meta-analysis. J Diabetes 2016;8(3):363-77.

[6] Leng GC, Fowkes FG. The edinburgh claudication questionnaire: an improved version of the WHO/Rose questionnaire for use in epidemiological surveys. J Clin Epidemiol 1992;45(10):1101-9.

[7] Taylor-Piliae RE, Fair JM, Varady AN, et al. Ankle brachial index screening in asymptomatic older adults. Am Heart J 2011;161(5):979-85.

[8] Nead KT, Cooke JP, Olin JW, et al. Alternative anklebrachial index method identifies additional at-risk individuals. J Am Coll Cardiol 2013;62(6):553-9.

[9] Aboyans V, Criqui MH, Abraham P, et al. Measurement and interpretation of the ankle-brachial index: a scientific statement from the American Heart Association. Circulation 2012;126(24):2890-909.

[10] Thiruvoipati T, Kielhorn CE, Armstrong EJ. Peripheral artery disease in patients with diabetes: epidemiology, mechanisms and outcomes. World J Diabetes 2015;6(7):961-9.

[11] Mourad JJ, Cacoub P, Collet JP, et al. Screening of unrecognized peripheral arterial disease (PAD) using ankle-brachial index in high cardiovascular risk patients free from symptomatic PAD. J Vasc Surg 2009;50(3):57280.

[12] Ness J, Aronow WS. Prevalence of coexistence of coronary artery disease, ischemic stroke and peripheral arterial disease in older persons, mean age 80 years, in an academic hospital-based geriatrics practice. J Am Geriatr Soc 1999;47(10):1255-6.

[13] Greenland P, Abrams J, Aurigemma GP, et al. Prevention conference $\mathrm{V}$ : beyond secondary prevention: identifying the high-risk patient for primary prevention: noninvasive tests of atherosclerotic burden: writing group III. Circulation 2000;101(1):E16-22.

[14] Murabito JM, Evans JC, Larson MG, et al. The anklebrachial index in the elderly and risk of stroke, coronary disease and death: the framingham study. Arch Intern Med 2003;163(16):1939-42.

[15] Prompers L, Apelqvist J, Apelqvist J, et al. High prevalence of ischaemia, infection and serious comorbidity in patients with diabetic foot disease in Europe. Baseline results from the Eurodiale study. Diabetologia 2007;50(1):18-25.

[16] Norgren L, Hiatt WR, Dormandy JA, et al. Inter-society consensus for the management of peripheral arterial disease (TASC II). J Vasc Surg 2007;45(Suppl S):S5-67.

[17] Schröder F, Diehm N, Kareem S, et al. A modified calculation of ankle-brachial pressure index is far more sensitive in the detection of peripheral arterial disease. J Vasc Surg 2006;44(3):531-6.

[18] Niazi K, Khan TH, Easley KA. Diagnostic utility of the two methods of ankle brachial index in the detection of peripheral arterial disease of lower extremities. Catheter Cardiovasc Interv 2006;68(5):788-92. 